\title{
Paramagnetic Meissner Effect in Multiply-Connected Superconductors
}

\author{
A. P. Nielsent, A. B. Cawthorneđ, P. Barbara, F. C. Wellstood, C. J. Lobb \\ Center for Superconductivity Research, University of Maryland, College Park, MD 20742 \\ R. S. Newrock \\ Department of Physics, University of Cincinnati, Cincinnati, OH 45421 \\ M. G. Forrester \\ Northrop Grumman Corporation, 1310 Beulah Rd, Pittsburgh, PA 15235
}

(October 23, 2018)

\begin{abstract}
We have measured a paramagnetic Meissner effect in $\mathrm{Nb}-\mathrm{Al}_{2} \mathrm{O}_{3}-\mathrm{Nb}$ Josephson junction arrays using a scanning SQUID microscope. The arrays exhibit diamagnetism for some cooling fields and paramagnetism for other cooling fields. The measured mean magnetization is always small, $\left\langle\Phi_{\text {tot }}-\Phi_{\text {ext }}\right\rangle<0.3 \Phi_{0}$ (in terms of flux per unit cell of the array, where $\Phi_{0}$ is the flux quantum) for the range of cooling fields investigated $\left(-12 \Phi_{0}\right.$ to $\left.12 \Phi_{0}\right)$. We demonstrate that a new model of magnetic screening, valid for multiply-connected superconductors, reproduces all of the essential features of paramagnetism that we observe and that no exotic mechanism, such as $d$-wave superconductivity, is needed for paramagnetism.
\end{abstract}

PACS numbers: 74.25.Ha, 74.20.-z, 74.50.+r, 75.20.-g

A paramagnetic response was first observed in BSCCOH and sybsequently reported in many other high $T_{c}$ materials. 0 This response is in striking contrast to the diamagnetic response which is a hallmark of superconductivity. It has been argued that the paramagnetic Meissner effect (PME) resultsfrom a $d$-wave symmetry in the BSSCQ prder parameter d The observation of PME in niobium 9 and aluminum, 10 known to be $s$-wave superconductors, questioned the validity of the $d$-wave argument.

There are several proposed theories for the PME, although no experiment has been yet able to provide conclusive evidence for one or another; and there is disagreement as to the validity of the $d$-wave explanation.11 In high $T_{c}$ materials one possible explanation is the existence of $\pi$-junctions which result from the misalignment of grains in materials with $d$-wave symmetry.6 In the conventional materials, which are known to be $s$-wave, PME has been attributed to different mechanisms causing nonequilibrium flux configurations. A nonequilibrium flux state may result from flux-compression, 12 the similar giant vortex state, 1314 pinning randomness, or a surface barrier at the edge of the sample.15

To create a controlled experiment we have chosen to look at Josephson-junction arrays using a scanning SQUID microscope (SSM), using a technique similar to that of Kirtley et al.16 The SSM provides spatially resolved magnetization images of the sample. Arrays eliminate many of the complications in studying the underlying cause of the PME. Since our arrays are lithographically fabricated from $\mathrm{Nb}$ and $\mathrm{Al}_{2} \mathrm{O}_{3}$, we know that there are neither $\pi$-junctions nor significant randomness. Additionally, our sample is uniformly attached to the thermal bath, eliminating thermal gradients across the sample during cooling. We find that arrays can be either paramagnetic or diamagnetic depending upon the cool- ing field. Additionally, we propose a new model, valid for multiply-connected supercondutors, which predicts a paramagnetic response and agrees with our scanning SQUID measurements.

The arrays consist of a square array of niobium crosses in two layers, with a unit cell size of $a=46 \mu \mathrm{m}$. Junctions are formed at the cross overlap. The calculated selfinductance of each loop of four junctions is $L=64 \mathrm{pH}$ and the measured critical current density is $J_{c}=600 \mathrm{~A} / \mathrm{cm}^{2}$ at $4.2 \mathrm{~K}$, with a junction area of $5 \times 5 \mu \mathrm{m}^{2}$. The entire array consists of $30 \times 100$ junctions. The unit-cell dimensions compare to the typical grain size seen in BSCCO samplest.16 which exhibit PME, to which similar fields are applied. The array is placed in an SSM sample stage, 17 sitting at the end of a sapphire rod of $10 \mathrm{~mm}$ diameter, around which a solenoid has been wound. Before any data is collected we first remove any flux trapped within the pick uploop of the SQUID to avoid interaction with the sample.18 Additionally, we measure and correct for any background field present.

We cooled our arrays to $4.2 \mathrm{~K}$ in fixed external magnetic field, and then used the SSM to measure the magnetic flux threading the SQUID from the sample, $\Phi_{\text {tot }}$, as a function of position. Our spatial resolution was limited by our SQUID-sample separation distance, which we estimate to be between 40 and $60 \mu \mathrm{m}$. Data were taken every $5 \mu \mathrm{m}$ in the $x$ direction and every $50 \mu \mathrm{m}$ in the $y$ direction, and converted from flux through the SQUID to normalized flux per unit cell. We measure the external flux, $\Phi_{\text {ext }}$, directly by warming the sample above $T_{c}$ and imaging. From the data we calculate both a local normalized magnetization $\left(\Phi_{\text {tot }}-\Phi_{\text {ext }}\right) / \Phi_{0}$ and an average overall magnetization $\left\langle\Phi_{\text {tot }}-\Phi_{\text {ext }}\right\rangle / \Phi_{0} .19$ We - were unable to resolve individual unit cells in the array, 20 but we were still able to resolve large scale flux distributions.

Our measurement and analysis proceeded in a fash- 
ion similar to Ref. 16. We first make an image of the magnetization of the array (a typical image is shown in Fig. 1(a)). We then make a magnetization histogram and compute the mean magnetization. Fig. Il(b) shows the magnetization histogram generated from the image in Fig. 11(a). The histogram is computed by looking at the lower third of the array and ceynting the number of points at a given magnetization.21 We find that the array may be either diamagnetic or paramagnetic, but surprisingly, appears to be preferentially paramagnetic. Additionally, we always observe diamagnetic screening currents around the outside edges of the sample. The array shown in Fig. 11(a) was cooled in an external field of $4.8 \Phi_{0}$ and clearly is paramagnetic (i.e. it is darker than the background). We find a mean paramagnetic response of $\left\langle\Phi_{\text {tot }}-\Phi_{\text {ext }}\right\rangle=(0.063 \pm 0.005) \Phi_{0}$ for this cooling field.

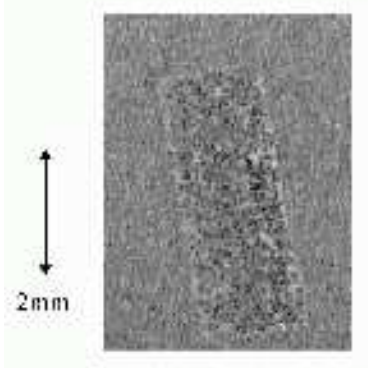

(a)



(hi)
FIG. 1. (a) Representative paramagnetic magnetization image for the array, with an external cooling field of $4.8 \Phi_{0}$. The color scale runs from $-0.6 \Phi_{0}$ (white) to $0.6 \Phi_{0}$ (black). (b) Magnetization histogram generated from the paramagnetic image.

The data plotted in Fig. 2 show the mean magnetization versus external cooling field. Each data point represents a single field-cooled average measurement. The error in the mean values plotted, such as discussed above, is less than the size of each data point as drawn. The sign of the magnetization is reproducible, and the magnitude is reproducible to within a factor of two when the sample is warmed above $10 \mathrm{~K}$ and recooled in the same field, indicating that our external field is consistent to within $\pm 0.05 \Phi_{0}$ The external field has a spatial variation of $\pm 0.5 \Phi_{0}$, which is limited by the gradient of the background field in our probe. Although there is some variation from one cooling to the next in the actual magnetic images for the same external field, the paramagnetism is remarkably reproducible.

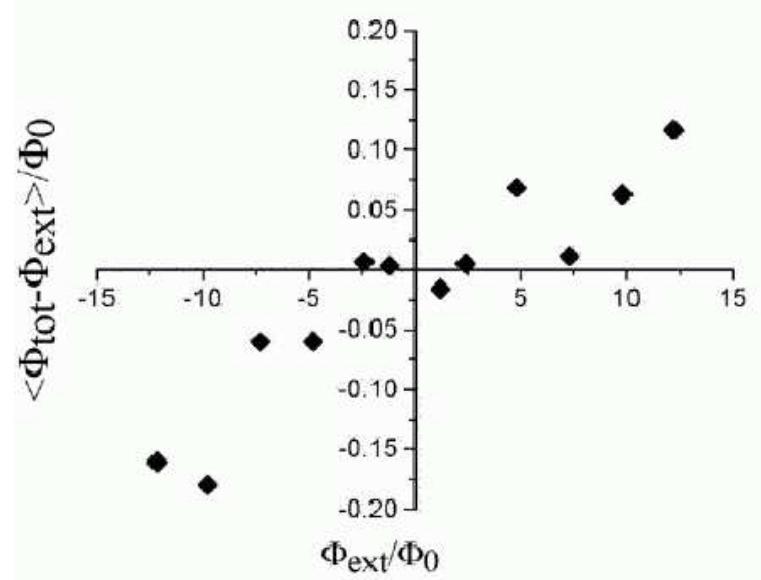

FIG. 2. Measured mean magnetization of the array $\left\langle\Phi_{\text {tot }}-\Phi_{\text {ext }}\right\rangle / \Phi_{0}$, plotted versus cooling field, $\Phi_{\text {ext }} / \Phi_{0}$. The error for each data point is less than the size of the plotted symbols.

We may consider a single loop of the array as a jumping off point for modeling the entire array's magnetization. Originally used to describe the observed magnetization of a single bop with a single Josephson-junction,22 Sigrist and Rice 6 later used this model to argue that $\pi$-junctions could cause PME in $d$-wave superconductors. The singleloop model gives a relationship between the external flux applied to a four-junction loop and the total measured flux

$$
\Phi_{\text {tot }}=\Phi_{\text {ext }}+L I_{c} \sin \left(\frac{\pi}{2} n-\frac{\pi}{2} \frac{\Phi_{\text {tot }}}{\Phi_{0}}\right) .
$$

Here $I_{c}$ is the critical current of a single junction. For all values of $\frac{L I_{c}}{\Phi_{0}}>\frac{2}{\pi}, \Phi_{\text {tot }}$ is a multi-valued function of $\Phi_{\text {ext }}$ depending on an integer $n$. We note here that Ref. 6 used a model with a $\frac{L I_{c}}{\Phi_{0}}$ slightly greater than one to demonstrate the absence of potential paramagnetism for a single loop with a single junction. Had they used a value of $\frac{L I_{c}}{\Phi_{0}} \gg 1$ they would have noticed the potential for paramagnetism even in the absence of external flux, although such a situation is not energetically favorable. For a four junction loop, $\beta_{\ell}=2 \pi \frac{L I_{c}}{\Phi_{0}}=30$ allows for the possibility of paramagnetism (or diamagnetism) at $\Phi_{\text {ext }}=0$.

Computing the Gibbs free energy for the various possible solutions to (1) we find that for $m<\frac{\Phi_{\text {ext }}}{\Phi_{0}}<m+\frac{1}{2}$ the single loop will be diamagnetic and for $m+\frac{1}{2}<\frac{\Phi_{\text {ext }}}{\Phi_{0}}<$ $m+1$ will be paramagnetic, where $m$ is any integer. If one applies a positive offset flux $\Phi_{\text {off }}$ to the four-junction loop (i.e. the total applied flux is $\Phi_{\text {ext }}+\Phi_{\text {off }}$ ) the solutions $\Phi_{\text {tot }}-\Phi_{\text {ext }}$ vs. $\Phi_{\text {ext }}$ become more paramagnetic and conversely if $\Phi_{\text {off }}<0$ the solutions become more diamagnetic. If, e.g., a simple diamagnetic screening current flows around the outside edge of the superconductor (negative offset), the measured magnetization will be more diamagnetic.

A conventional superconductor is a perfect diamagnet: An applied flux causes screening currents to flow around 
the outside. In an ideal array of Josephson junctions, one may decompose this edge current, $I$, into loop currents of magnitude $I$, flowing around each plaquette, as shown in Fig. 3(a). In a real array, this is not likely to be the complete picture. The currents will flow only within one penetration depth in the superconductor. For our array, the penetration depth is $900 \AA$ while the wire size is $10 \mu \mathrm{m}$ so that the interior loop currents do not cancel. This means that the array must create an energy $\frac{1}{2} L I^{2}$ for each plaquette in order to generate a screening current $I$ for the array. In an overlap geometry, the currents through the Josephson-junctions are uniform, so the currents through the junctions still cancel and we do not have any increase in the total Josephson energy, $E_{J}$. For an array of $N \times N$ junctions Fig. 3(a) costs a total energy of

$$
E_{a}=\frac{1}{2} L I^{2} N^{2}+4 N(1-\cos \gamma) E_{J}
$$

where $\gamma$ is the gauge invariant phase difference.

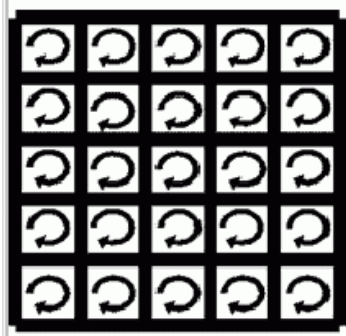

(a)

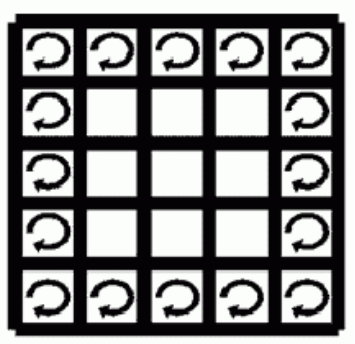

(b)
FIG. 3. Mechanisms for generating the diamagnetic screening current around a multiply connected superconducting sample: (a) The conventional picture, where each loop of the sample generates a current. (b) Our new picture, where only the exterior plaquettes create a screening current. This creates a diamagnetic current on the outside of the sample, and a paramagnetic current just inside the sample.

We propose that the array actually screens in a much different fashion. The array may generate a diamagnetic screening current around the outside perimeter if each exterior plaquette maintains a screening current. as shown schematically in Fig. 3(b). In addition to the diamagnetic screening current, we then also have a paramagnetic current, of the same magnitude, flowing just inside the array. The array must generate the $\frac{1}{2} L I^{2}$ energy only for each exterior plaquette, so the energy cost to the array in this case is

$$
E_{b}=\frac{1}{2} L I^{2}(4 N)+\{4 N+4(N-1)\}(1-\cos \gamma) E_{J} .
$$

It is clear from (22) and (3) that $E_{a} \propto N^{2}$ while $E_{b} \propto N$ so that for a large enough array, the conventional picture of screening will not be energetically favorable. Fig. 岛(b) has lower energy than Fig. 3(a) when, assuming $\gamma$ is proportional to $\Phi_{\text {tot }}$,

$$
\beta_{\ell}>\frac{4 N-1}{N^{2}-4 N}, \forall N>2
$$

For our array the criteria is easily satisfied and we expect screening to take place as in Fig. 3 (b).

In Fig. 3(a) we can treat the outer ring of Josephsonjunctions as a single loop of $4 N$ junctions. This loop will screen up to $\frac{L I_{c}}{\Phi_{0}}=4.8$ flux quanta from the interior of the array, when $I_{c}$ is flowing around the exterior of the array. In Fig. 自(b) each exterior plaquette can screen up to $\frac{L I_{c}}{\Phi_{0}}$ flux quanta from itself. This is a much larger absolute field than in the conventional case and the difference can be easily observed experimentally. We have observed, that flux will not penetrate the array until the external flux exceeds at least $4.0 \Phi_{0}$ per unit cell. Additionally, ac susceptibility work demonstrated that little effect was seen until the amplitude of the driving ac flux exceeded $4.6 \Phi_{0}$ per unit cell of the array 23

The interior plaquettes see the external field, a diamagnetic flux from the extreme outside loop of the array, and a paramagnetic flux from just inside the array. Because the loop generating the paramagnetic flux is closer, the interior loops will see a net paramagnetic flux in addition to the external field. Considered individually, the interior plaquettes obey a modified Eq. (11),

$$
\Phi_{\mathrm{tot}}=\Phi_{\mathrm{ext}}+\Phi_{\mathrm{scr}}+L I_{c} \sin \left(\frac{\pi}{2} n-\frac{\pi}{2} \frac{\Phi_{\mathrm{tot}}}{\Phi_{0}}\right)
$$

where $\Phi_{\text {scr }}$ is the flux seen by the inner plaquette due to the screening currents produced by the exterior plaquettes. When $\Phi_{\text {scr }}>0$ the interior plaquette sees a paramagnetic offset and its magnetization will be more paramagnetic. This causes the array to be paramagnetic more often than diamagnetic in agreement with experiment.

If we consider only the mutual inductance between a test plaquette in the interior of the array and the exterior plaquettes which provide the screening, we can make a crude estimate of the paramagnetic response. The maximum current that the junctions can sustain is the critical current, $I_{c}$; this sets the upper bound for the magnetization offset. We have numerically computed the flux induced from the screening currents in Fig. 3(b) onto the interior plaquettes for our $30 \times 100$ junction array. When the screening currents are $I_{c}$, the maximum flux is induced, and the minimum $\Phi_{\text {scr }}$ we compute is $0.15 \Phi_{0}$ per unit cell, near the center of the array. This means that the center plaquette should have its magnetization shifted upward by $0.15 \Phi_{0}$. Plaquettes nearer to the exterior see $\Phi_{\text {scr }}$ values as large as several $\Phi_{0}$. Additionally, we compute an average flux induced into the interior of the array to be $\Phi_{\mathrm{scr}}=0.277 \Phi_{0}$ per unit cell. Considering the crudeness of the model, this is remarkable agreement with our measured magnetization (see Fig. 2).

We expect that the screening current will increase monotonically with the external flux, up to $I_{c}$, and therefore expect that the paramagnetic offset in the interior plaquette magnetization will increase as the external flux 
increases. This is also consistent with our observations in Fig. 2.

The model described here does not account for many features of the real array. We have not accounted for variations in the Josephson-junction parameters and we have ignored the mutualinduction between interior plaquettes. Phillips et al.24 showed the importance of including the mutual inductance matrix when computing array magnetization. Chandran has, in the overdamped junction limit, used a model including the full inductance matrix and computed paramagnetic moment in a Josephson-junction array.25 Unfortunately, our arrays are highly underdamped, which makes a model utilizing the full-inductance and dynamics much more computationally difficult.26 The simplicity of our model does not describe all of the features that we observe in our array. The observed flux pattern is very complicated, with both paramagnetic and diamagnetic regions.

We have shown that in a multiply connected superconductor such as our array, that the lowest energy configuration that provides diamagnetic screening will also tend to make the interior of the array paramagnetic. Furthermore, the predictions of this model are consistent with our measurements, that the array is more often paramagnetic than diamagnetic. We believe that the paramagnetism in Josephson-junction arrays is best described by the effects detailed above.

We would like to thank J. R. Kirtley, M. Sigrist and M. Chandran for useful communications. Additionally we would like to thank R. L. Greene for his critical reading of our manuscript. We acknowledge support from AFOSR Grant F49620-98-1-0072, NSF Grant DMR-9732800 and NSF Grant DMR-9801825.

* Electronic address: anielsen@squid.umd.edu.

$\dagger$ Present address: Neocera, Inc., 10000 Virginia Manor Road, Beltsville, MD 20705, USA.

${ }^{1}$ W. Braunisch, N. Knauf, V. Kataev, A. Grutz, A. Kock, B. Roden, D. Khomskii, and D. Wohlleben, Phys. Rev. Lett. 68, 1908 (1992) and W. Braunisch, N. Knauf, B. Bauer, A. Kock, A. Becker, B. Freitag, A. Grutz, V. Kataev, S. Neuhausen, B. roden, D. Khomskii, and D. Wohlleben, Phys. Rev. B 48, 4030 (1993).

2 B. Schliepe, M. Stindtmann, I. Nikolic, and K. Baberschke, Phys. Rev. B 47, 8331 (1993).

${ }^{3}$ S. Riedling, G. Brauchle, R. Lucht, K. Rohberg, and H. v. Lohneysen, Phys. Rev. B 49, 13283 (1994).

${ }^{4}$ U. Onbasli, Y. T. Wang, A. Naziripour, R. Tello, W. Kiehl, and A. M. Hermann, Phys. Stat. Sol. B 194, 371 (1996).

${ }^{5}$ G. S. Okram, D. T. Adroja, B. D. Padalia, O. Prakash, and P. A. J. de Groot, J. Phys.: Cond. Mat. 9, L525 (1997).

${ }^{6}$ M. Sigrist and T. M. Rice, J. Phys. Soc. Jpn. 61, 4283 (1992).

${ }^{7}$ D. J. Thompson, M. S. M. Minhaj, L. E. Wenger, and J. T.
Chen, Phys. Rev. Lett. 75, 529 (1995).

${ }^{8}$ P. Kostic, A. P. Paulikas, U. Welp, V. R. Todt, C. Gu, U. Geiser, J. M. Williams, K. D. Carlson, and R. A. Klemm, Phys. Rev. B 53, 791 (1996).

${ }^{9}$ A. Terentiev, D. B. Watkins, and L. E. D. Long, Phys. Rev. B 60, R761 (1999).

${ }^{10}$ A. K. Geim, S. V. Dubonos, J. G. S. Lok, M. Henini, and J. C. Mann, Nature 396, 144 (1998).

11 This disagreement is highlighted by the comments following the publication of Ref. G in T. M. Rice and M. Sigrist, Phys. Rev. B 55, 14647 (1997) and P. Kostic, B. Veal, A. P. Paulikas, U. Welp, V. R. Todt, C. Gu, U. Geiser, J. M. Williams, K. D. Carlson, and R. A. Klemm, Phys. Rev. B 55, 14649 (1997).

12 A. E. Koshelev and A. I. Larkin, Phys. Rev. B 52, 13559 (1995).

${ }^{13}$ V. V. Moschalkov, X. G. Qiu, and V. Bruyndoncx, Phys. Rev. B 55, 11793 (1999).

${ }^{14}$ P. S. Deo, V. A. Schweigert, F. M. Peeters, and A. K. Geim, Phys. Rev. Lett. 79, 4653 (1997).

${ }^{15}$ P. S. Deo, V. A. Schweigert, and F. M. Peeters, Phys. Rev. B 59, 6039 (1999).

${ }^{16}$ J. R. Kirtley, A. C. Mota, M. Sigrist, and T. M. Rice, J. Phys.: Cond. Mat. 10, L97 (1998).

${ }^{17}$ R. C. Black, A. Mathai, F. C. Wellstood, E. Dantsker, A. H. Miklich, D. T. Nemeth, J. J. Kingston, and J. Clarke, Appl. Phys. Lett. 62, 2128 (1993).

18 A. Mathai, D. Song, Y. Gim, and F. C. Wellstood, Appl. Phys. Lett. 61, 598 (1992).

${ }^{19} \Phi_{\text {ext }}=H a^{2}$ and $\Phi_{\text {tot }}=B a^{2}$ so that $\left(\Phi_{\text {tot }}-\Phi_{\text {ext }}\right)=4 \pi M a^{2}$ where $H=B-4 \pi M$ and $B$ is the magnetic induction, $H$ is the magnetic field and $M$ is the magnetization, in Gaussian units.

20 The size of our SQUID is $10 \times 10 \mu \mathrm{m}^{2}$, while the separation between the SQUID and sample is between 40 and $60 \mu \mathrm{m}$. The SQUID-sample separation limits the spatial resolution we can achieve to roughly $50 \mu \mathrm{m}$. Because of this, and the design of our sample stage, it was not feasible to scan at a higher than $50 \mu \mathrm{m}$ resolution in the $y$ direction. Because we do not undersample the array the average we quote for the magnetization of the array will be correct.

${ }^{21}$ Scratches were accidentally introduced into the array in situ. In order to ignore complications arising from these scratches in the analysis of the data we have chosen to only analyze the unblemished region of the array.

22 A. H. Silver and J. E. Zimmerman, Phys. Rev. 157, 317 (1967).

${ }^{23}$ F. M. Araujo-Moreira, P. Barbara, A. B. Cawthorne, and C. J. Lobb, Phys. Rev. Lett. 78, 4625 (1997) and P. Barbara, F. M. Araujo-Moreira, A. B. Cawthorne, and C. J. Lobb, Phys. Rev. B 60, 7489 (1999).

${ }^{24}$ J. R. Phillips, H. S. J. van der Zant, J. White, and T. P. Orlando, Phys. Rev. B 47, 5219 (1993).

${ }^{25}$ M. Chandran, Phys. Rev. B 56, 6169 (1997).

${ }^{26}$ We have partially addressed this issue by considering the effects on one plaquette of its neighbors in a mean field approximation and discovered that, surprisingly, the paramagetism of the array is enhanced a small amount. 